\title{
Research on On-line Tool Wear Monitoring Technology Based on GPR
}

\author{
Shan Fan ${ }^{1, a}$, Yi Huang ${ }^{1, b}$ and Haixia Zeng ${ }^{1, c^{*}}$ \\ ${ }^{1}$ Mechanical \& Electronic Engineering Division, WenHua College, Wuhan, Hubei, 430074, China
}

\begin{abstract}
At present, many kinds of sensors are used for on-line monitoring of cutting process, tool identification and timely replacement. However, most of the original monitoring signals extracted from the cutting process are time series signals, which contain too much process noise. As the signal noise is relatively low, it is difficult to establish a direct relationship with the tool wear. Therefore, how to obtain the effective information from the online monitoring signal and extract the characteristics that can directly reflect the tool wear from the complex original signal, so as to establish an effective and reliable tool wear monitoring system, is the key and difficult problem in the research of the online monitoring technology of tool wear. Firstly, an experimental platform based on the force sensor for on-line monitoring of tool wear was built, and the signal obtained by the force sensor was used to monitor the tool wear, and the feature information was extracted and fused. The innovation of the project lies in the use of Gaussian process regression (GPR) method to predict the tool wear, the use of feature dimensional rise technology, to reduce the impact of noise, on the premise of ensuring the prediction accuracy, improve the confidence interval of GPR prediction results, improve the stability and reliability of the monitoring process.
\end{abstract}

\section{Introduction}

Since the 1970s, many scholars at home and abroad have done a lot of research on the monitoring technology or system of tool wear or damage, put forward various monitoring methods, and designed relevant monitoring instruments or systems. High productivity and high machining precision have become an important benchmark for a country's manufacturing level in the era of intelligent manufacturing. During the machining and manufacturing process, the state of tool wear is an important factor affecting the surface quality and dimensional accuracy of the work piece to be machined. Excessive use of severely worn tools will lead to the scrap of damaged tool parts and the increase of unexpected downtime, resulting in a waste of resources. Tool replacement mainly depends on the personal experience of skilled workers to determine the wear state of the tool, but with the increase of labor costs, this method is clearly uneconomical. In addition, the tool wear is a complex phenomenon, through the non-linear way to show, so sometimes there will be misjudgment of the tool wear state. It is of great significance to develop an on-line monitoring system for tool wear and to monitor the wear state of the tool in real time. The following aspects can be implemented; (1) Establish a more reasonable and effective tool changing strategy to extend the service life of the tool as much as possible; (2) Reduce the downtime caused by tool breakage to ensure smooth processing and increase productivity; (3) According to the wear condition

ae-mail: fanshan05@163.com, be-mail: h13971359810@163.com

*Corresponding author's ce-mail: zenghaixia@foxmail.com of the cutting tools, the corresponding error compensation is carried out to ensure the machining accuracy and surface quality of the workpiece. In addition, accurate evaluation of tool wear state is of great significance to ensure the smooth progress of manufacturing process and high quality product quality. Tool wear is one of the key factors to ensure the reliability and stability of manufacturing system, because excessive tool wear will lead to sharp increase in cutting force, and even cause machine chatter.[1] With the implementation of made-inChina 2025 plan, modern manufacturing systems are becoming more and more complex, with higher requirements for reliability and stability, and monitoring systems are required to provide early warning of tool wear. In the machining of $\mathrm{CNC}$ machine tools, the factors that affect the machining quality include the machine tool system error, mechanical deformation error, machining method error and tool wear error, among which the machining error caused by tool wear is one of the main factors. Therefore, it is of great theoretical significance and application value to study the rule of tool wear and compensate the cutting part according to the wear rule curve.

\section{Cutting tool wear test}

In order to study the relationship between cutting force and tool wear, the turning tool wear experiment was carried out in this study to establish tool wear prediction model. The equipment and instruments for the turning tool wear experiment are shown in Figure 1.The model of the 
machine tool is DMTG-CW6163E; The workpiece is 50\# normalizing steel (HB160 197), the cutting diameter is $200 \sim 110 \mathrm{~mm}$, and the cutting length is $400 \mathrm{~mm}$. The carbide indexable blade and the clamped blade produced by Sandvik are used respectively. The blade model is CNMG120408-PM, and the blade model is PCLNR $2525 \mathrm{M} 12$. The cutting force acquisition system, composed of charge amplifier of dynamometer and data acquisition card, is used to collect the three-way orthogonal cutting forces in the cutting process,, and can display the changes of cutting forces in real time through software DynoWare. The position relationship between the turning tool and the dynamometer is shown in Figure1.
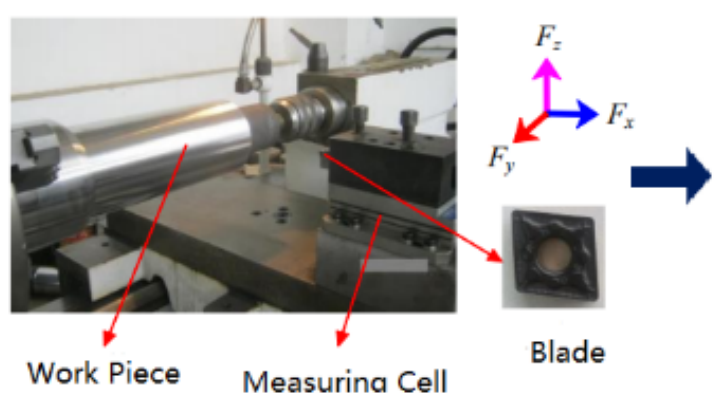

\section{Charae-amplifier}

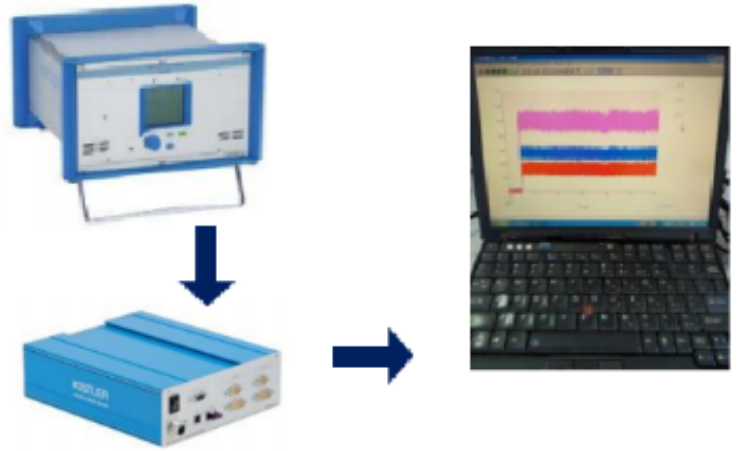

Data Acquisition Card

Figure 1.Experimental apparatus and instruments

In this study, data acquisition mainly consists of two parts: one is the on-line measurement of cutting force, whose sampling frequency is $20 \mathrm{kHz}$. The cutting parameters of the turning tool wear experiment are shown in Table 1. The first four groups of experiments are repeated twice, while the last two groups are only repeated once. The first four groups of experiments are repeated twice, while the last two groups are only repeated once. Each wear value corresponds to a set of data of three-way cutting force. There are 20,000 data points of one-way cutting force collected every second, which is a huge amount of data and cannot directly establish the relationship between cutting force and tool wear. Therefore, it is necessary to extract the signal characteristics from the cutting force signal in a certain period of time and vectorize the cutting force signal in order to reflect the change of tool wear intuitively.

Table 1. Cutting parameters of turning experiment

\begin{tabular}{lcccc}
\hline Test No. Cutting velocity $\mathrm{Vc}(\mathrm{m} / \mathrm{min})$ & Feed $\mathrm{f}(\mathrm{mm} / \mathrm{r})$ & Cutting depth $\mathrm{ap}(\mathrm{mm})$ & Times of repetition \\
\hline 1 & 300 & 0.2 & 1 & 2 \\
2 & 300 & 0.2 & 2 & 2 \\
3 & 300 & 0.3 & 1 & 2 \\
4 & 300 & 0.3 & 2 & 2 \\
5 & 300 & 0.4 & 1 & 1 \\
6 & 300 & 0.4 & 2 & 1 \\
\hline
\end{tabular}

\section{Extraction and fusion of monitoring features}

Usually, it is difficult to directly establish the relationship between the monitoring signal and tool wear due to the large amount of data and low signal noise, so it is necessary to extract the signal characteristics that can objectively reflect the tool wear state from the monitoring signal. For the turning tool wear experiment, a total of 48 monitoring features were extracted, including 18 timedomain features, 6 frequency-domain features, and 24 wavelet domain features. The feature vector composed of 3 cutting parameters and 48 monitoring features is taken as the input of tool wear monitoring model based on GPR.
In the turning experiment, a total of 215 data files were collected and recorded. One data file corresponds to a cutting process, and a total of 430 data samples containing feature vectors were obtained, which were equally divided into non-overlapping training sets and test sets. These training sets and test sets contain a complete tool wear process (from new to broken). The training set is used to train the tool wear monitoring model, and the test set is used to test the performance of the established tool wear model. Since the extracted features contain a large amount of process noise or redundancy, which will affect the training time or predictive performance of the monitoring model, it is necessary to carry out feature fusion to eliminate noise and reduce its negative effects. At this point, the feature vector composed of the fusion feature 
and the corresponding cutting parameters should be taken as the input of the monitoring model. For the turning tool wear experiment, the $\mathrm{kPCa}$-based characteristic dimension raising technology is adopted in this paper to reduce the influence of noise, improve the confidence interval of GPR prediction results and improve the stability of the monitoring process on the premise of ensuring the prediction accuracy.

\section{Tool wear prediction based on GPR}

A Gaussian distribution is a probability distribution describing a random variable (scalar vector) whose probability density (property) is determined entirely by means and variance. The Gaussian process is the extension of the Gaussian distribution in the low dimensional space to the high dimensional space. It is used to describe the function distribution and is an important random process. The tool wear prediction model based on GPR is

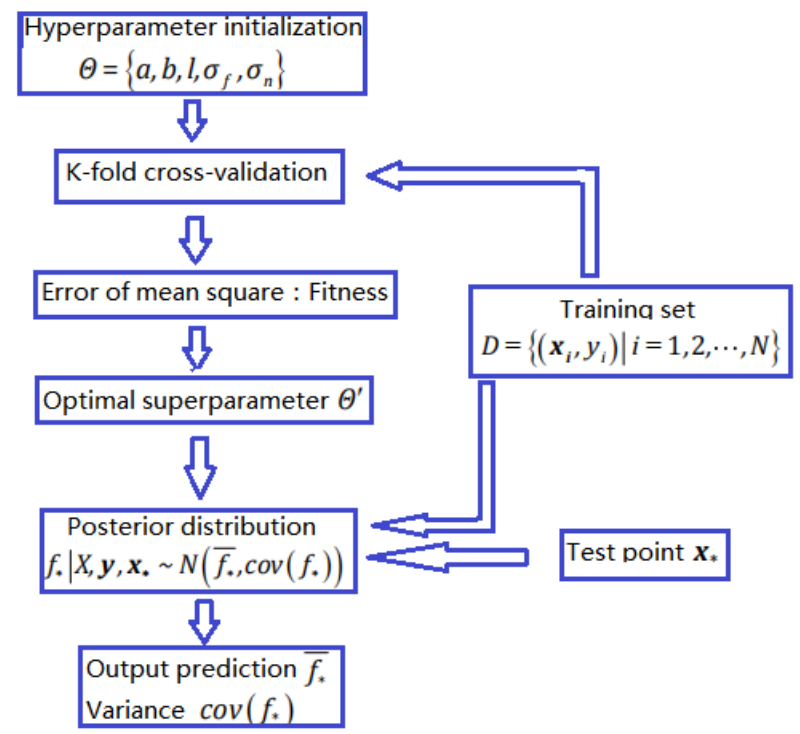

Figure 2.Parameter optimization of GPR model

\section{Conclusions}

In this chapter, a new tool wear assessment technique based on GPR model is studied to accurately monitor the wear width of tool back face during machining. The main work is summarized as follows:1) Since Gaussian noise can be quantitatively modeled in GPR model, the PREDICTION accuracy of GPR model is high and the confidence interval of prediction results can be provided.2) It has the advantages of high prediction accuracy, small calculation amount (CV time) and comparable Test time, etc., especially suitable for on-line monitoring of tool wear. Tool wear monitoring is a very complex technology with a broad application prospect, but no significant breakthrough has been made so far. For tool wear monitoring, domestic and foreign scholars do the research, also limited to the laboratory stage, the industrial promotion has not yet come into sight. It is feasible in constructed by fitting the target value and fusion characteristics. Generally speaking, the prediction accuracy of GPR models is almost unaffected by Gaussian noise, because they can be quantitatively modeled and integrated into the Gaussian process. Therefore, the GPR model has better prediction accuracy than other AI models, such as artificial neural network (ANN) and support vector machine (SVM). $n$ the GPR model, the superparameters are unknown and need to be inferred by conjugate gradient algorithm and given training set. The parameters of the given mean function are initialized to $\mathrm{a}=0$ and $\mathrm{b}=0$. The characteristic scale parameter of the covariance function is initialized to $\mathrm{L}=1$. The range of standard deviation of signal and noise is determined by trial and error: $\sigma_{f} \times \sigma_{n} \in[0.01,0.1] \times[0.0001,0.001]$.According to the fitness of GPR model, the appropriate initialization parameters are determined by using grid search and K-fold cross validation.

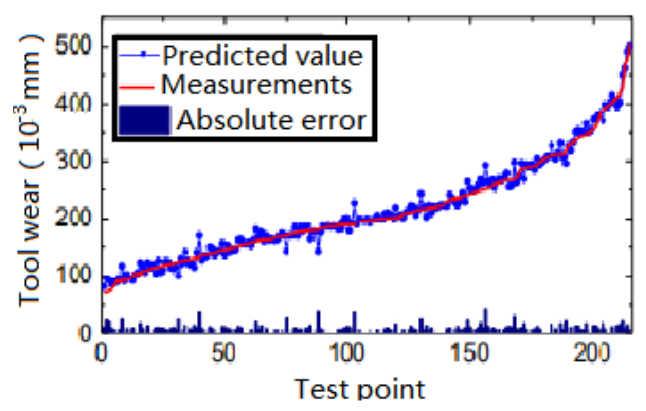

Figure 3.Predicting Outcomes of GPR model for tool wear monitoring

theory to accurately monitor the wear width of the tool's rear surface, but it is quite difficult to operate in practice, because there are many unknown factors that affect the prediction accuracy of the model at any time during the cutting process.

\section{Acknowledgments}

I gratefully acknowledge the financial support of Hubei Provincial Department of Education.(No. B2019304)

\section{References}

1. C. Zhang, H. Wei, X. Zhao, T. Liu, K. Zhang, A Gaussian process regression based hybrid approach for short-term wind speed prediction, Energy Conversion and Management 126 (2016) 1084-1092.

2. Y. Wang, B. Chaib-draa, KNN-based Kalman filter: An efficient and non-stationary method for Gaussian 
process regression, Knowledge-Based Systems 114 (2016) 148-155.

3. S.A. Aye, P.S. Heyns, An integrated Gaussian process regression for prediction of remaining useful life of slow speed bearings based on acoustic emission, Mechanical Systems and Signal Processing 84 (2017) 485-498.

4. Y. Liu, Y. Pan, D. Huang, Q. Wang, Fault prognosis of filamentous sludge bulking using an enhanced multi-output gaussian processes regression, Control Engineering Practice 62 (2017) 46-54. 